\title{
Stereotactic radiotherapy for the treatment of lung cancer with a giant left atrial tumor thrombus: A case report and literature review
}

\author{
YONG LI $^{1}$, JINRONG LOU ${ }^{2}$, SHUJUN QIU $^{1}$, YUTIAN GUO $^{1}$ and MIANSHUN PAN ${ }^{1}$ \\ ${ }^{1}$ Center of Radiation Oncology, Wujing Hospital, Shanghai 201103; ${ }^{2}$ Department of Cardiology, Jiading Central Hospital, \\ Shanghai 201800, P.R. China
}

Received February 13, 2015; Accepted December 16, 2015

DOI: $10.3892 / 01.2016 .4215$

\begin{abstract}
Lung cancer presenting with a giant atrial tumor thrombus is particularly rare. Surgical resection, aided by a cardiopulmonary bypass, is the standard treatment of choice if there is no distant metastasis. However, this form of surgery carries a high risk, with the subsequent patient prognosis being extremely poor. The current study describes the case of a 52-year-old man presenting with left lung squamous cell carcinoma that had extended into the left atrium. The patient was treated with stereotactic radiotherapy, and regarding the atrial disease, a complete response was achieved within 12 months. The present case demonstrates that stereotactic radiotherapy may be a beneficial palliative treatment for patients with stage IV lung cancer invading the left atrium.
\end{abstract}

\section{Introduction}

Advanced non-small cell lung cancer with associated atrial involvement is uncommon, occurring in $\sim 10 \%$ of patients presenting with bronchogenic carcinoma $(1,2)$. Patients with the disease usually possess circulatory and respiratory symptoms, and coughing, hemoptysis, dyspnea, weight loss and an extremely poor performance status are the typical associated features (3). Furthermore, an associated intra-atrial tumor thrombus is particularly rare, with tumor thrombi potentially leading to widespread systemic embolization and/or outflow tract obstruction (4-6). As a result, treatment is always warranted. If there is no presentation of distant metastasis, surgical resection is considered as the gold-standard therapy. However, chemotherapy may also be administered. Cases of non-small cell lung cancer with associated intra-atrial tumor thrombi are usually diagnosed using enhanced computed tomography (CT).

Correspondence to: Professor Mianshun Pan, Center of Radiation Oncology, Wujing Hospital, 380 Hongxu Road, Shanghai 201103, P.R. China

E-mail:panmianshun@126.com

Key words: lung cancer, atrium, tumor thrombus, stereotactic radiotherapy
Due to the rarity of this condition, incidence and mortality rates remain unknown, however, patient prognosis is extremely poor with an overall survival time of 4-17 months $(1,7)$. The present study reports the case of a patient with stage IV lung cancer with invasion into the left atrium, for which a complete response was achieved following treatment with stereotactic radiotherapy.

\section{Case report}

In April 2012, a 52-year-old man was admitted to the Center of Radiation Oncology, Wujing Hospital (Shanghai, China) presenting with a 3-month history of progressive dyspnea and coughing. The patient also had a history of hypertension and smoking. An enhanced CT scan (Optima CT660 FREEdom; GE Healthcare, Piscataway, NJ, USA) revealed a left hilar tumor wrapped around the left pulmonary artery and vein, along with a large mass in the left atrium (Fig. 1A). A transbronchial biopsy was performed. Subsequently, tissue was formalin-fixed (Shanghai Ziyi Reagent Factory, Shanghai, China) paraffin-embedded (Leica Microsystems, Ltd., Milton Keynes, UK) and cut into $4 \mu \mathrm{m}$ sections for hematoxylin and eosin (Baso Diagnostics Inc., Zhuhai, China) staining and immunohistochemistry. The biopsy revealed that the primary site was consistent with nests and trabecula of carcinoma cells proliferating with necrotic debris and intercellular bridges structures (Fig. 2A and B). For immunohistochemical analysis, the specimens were incubated with primary antibodies for $45 \mathrm{~min}$ at room temperature. Slides were then washed 4 times in Tris-Buffered Saline with Tween 20 (TBST) for $5 \mathrm{~min}$. The slides were incubated with horseradish peroxidase-conjugated polyclonal goat anti-rabbit secondary antibody (Dako REAL ${ }^{\mathrm{TM}}$ EnVision $^{\text {TM }}$ kit; ready to use; cat. no. K5007; Dako, Glostrup, Denmark) for $30 \mathrm{~min}$ at room temperature. The slides were then washed 3 times with TBST for $5 \mathrm{~min}$. Next, slides were washed with Dako REAL ${ }^{\mathrm{TM}}$ substrate buffer (Dako) and incubated with 3,3'-diaminobenzidine chromogen (Dako) for $10 \mathrm{~min}$ at room temperature. The slides were then washed 3 times with TBST followed by distilled water for $1 \mathrm{~min}$. Staining was visualized under a microscope (BX41; Olympus Corporation, Tokyo, Japan). Immunohistochemistry revealed positive staining for cytokeratin 5/6 (monoclonal anti-human mouse antibody; 1:50; cat. no. AM0101; Ascend 
A

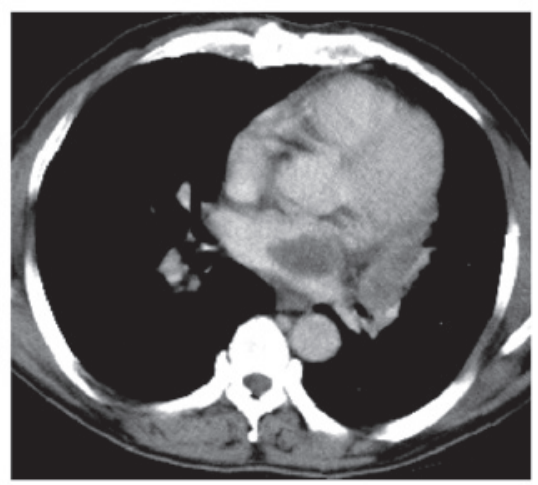

C

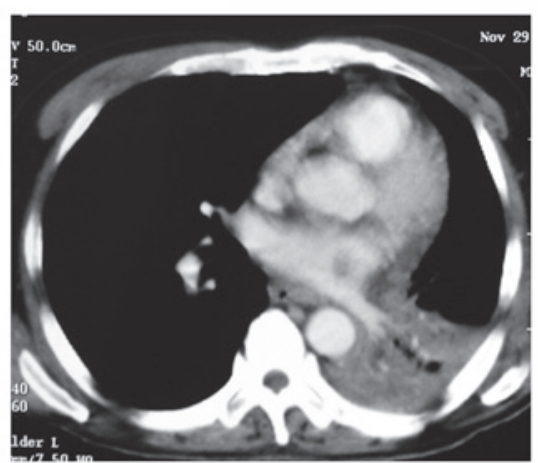

B
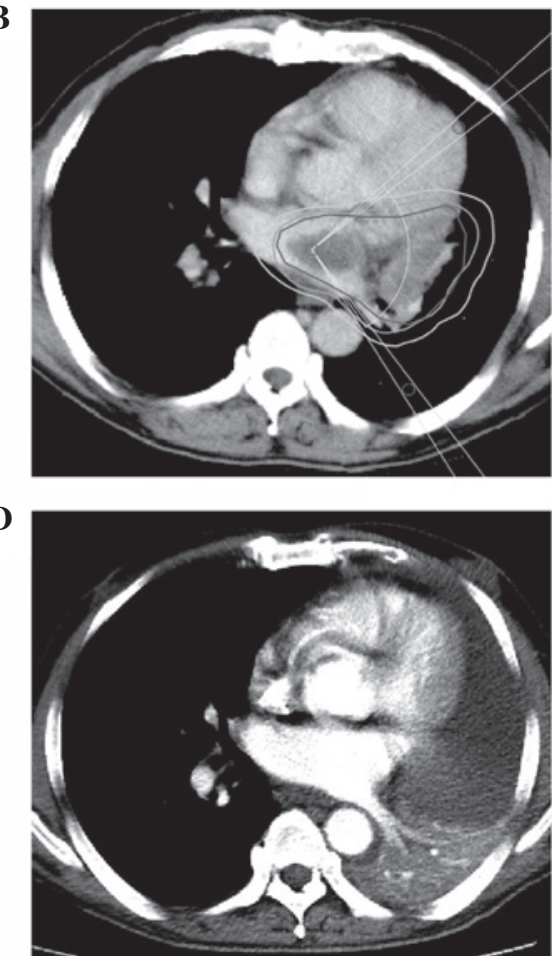

Figure 1. Intracardiac extension of the tumor at first presentation and post-treatment. (A) CT scan demonstrating a large left atrial tumor thrombus. (B) CT scan showing the isodose line of the radiotherapy planning system. (C) CT scan demonstrating a clear reduction of the left atrial tumor thrombus and the left lower pulmonary tuberculosis following 7 months of radiotherapy. (D) CT scan demonstrating that the tumor thrombus had disappeared following 12 months of radiotherapy. $\mathrm{CT}$, computed tomography.
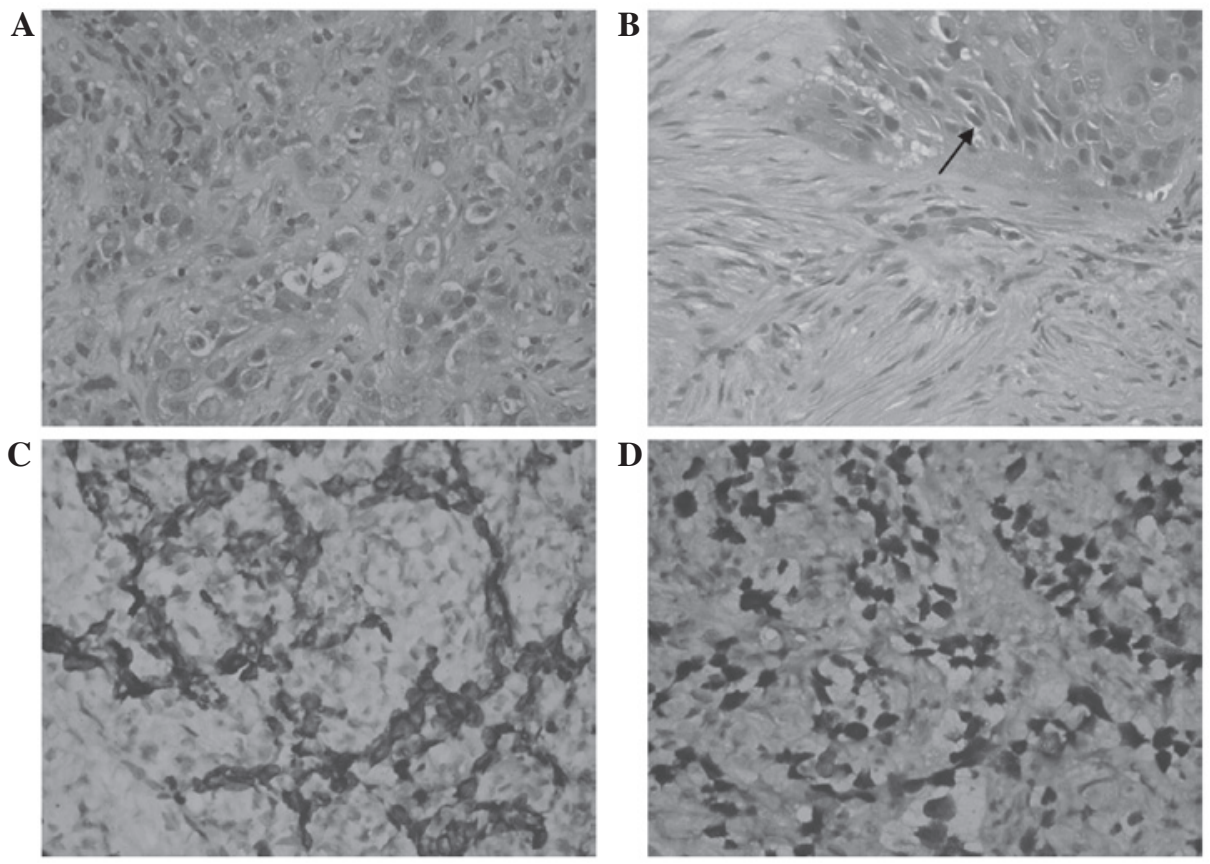

Figure 2. Histopathological analysis of bronchial biopsy specimens. (A) Hematoxylin and eosin staining revealing (A) poorly differentiated squamous cell carcinoma (magnification, x200) and (B) intercellular bridges (indicated by the black arrow) of squamous cell carcinoma (magnification, x200). (C) Immunohistochemical staining revealing positivity for (C) CK5/6 (magnification, x200) and (D) p40 (magnification, x200) within the areas of carcinoma nests.

Biotechnology Co., Ltd., Guangzhou, China) and p40 (monoclonal anti-human mouse antibody; 1:200; cat. no. 10035N; Ascend Biotechnology Co., Ltd.), which indicated poorly differentiated squamous cell carcinoma (Fig. 2C and D).
Positron emission tomography (PET; GE Discovery ST; GE Healthcare) exhibited intense ${ }^{18} \mathrm{~F}$-fluorodeoxyglucose uptake in the hilar tumor and the mass in the left atrium; additionally, a hot spot was located in the left adrenal gland (Fig. 3). 


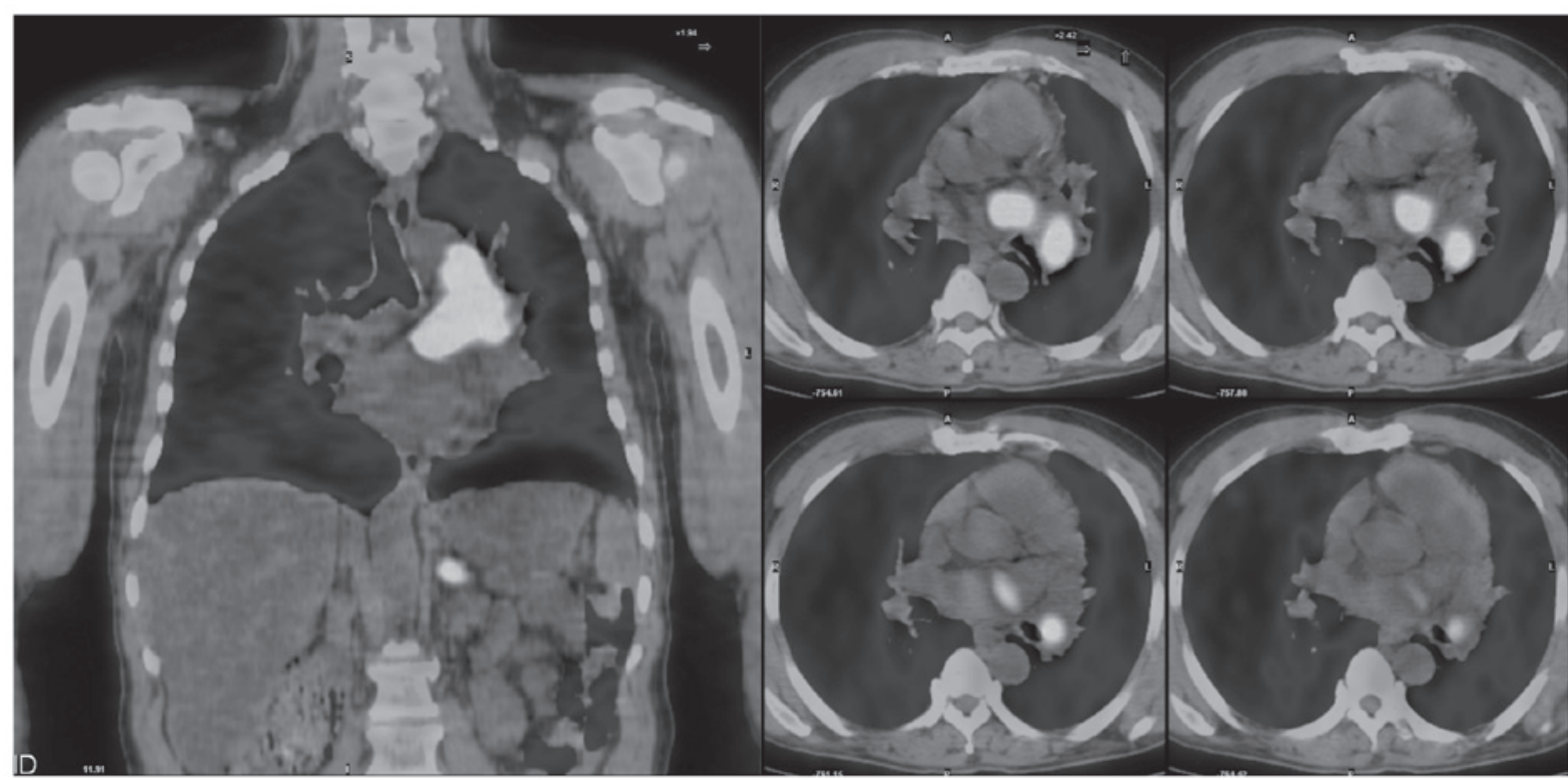

Figure 3. Positron emission tomography scans demonstrating intense ${ }^{18} \mathrm{~F}$-fluorodeoxyglucose uptake in the hilar mass and the left atrial tumor thrombus.

The patient was therefore diagnosed with T4N1M1 [American Joint Committee on Cancer stage IV (8)] lung squamous cell carcinoma with atrial tumor thrombus.

Due to the high risks of surgical resection and a patient history of refractory diabetes, it was agreed that stereotactic radiotherapy, via segmented gamma-ray therapy (SGS-I, Huiheng Medical, Shenzhen, China), would be performed on the two lesions. An internal target volume (ITV) of the tumor was segmented according to tumor motion based on a four-dimensional scan. A 6-mm margin was added to the ITV to form the planning target volume (PTV). A total dose of $40 \mathrm{~Gy}$ in 4-Gy fractions was delivered over 2 weeks to cover at least $95 \%$ of the PTV (Fig. 1B). The median doses for the lung and esophagus were 6.8 and $12.6 \mathrm{~Gy}$, respectively. The maximum dose administered to the spinal cord was $5.2 \mathrm{~Gy}$, and the $\mathrm{V}_{30}$ [volume (\%) receiving $30 \mathrm{~Gy}$ ] of the uninvolved heart was $20.8 \%$. Following a total of 2 months, a CT scan demonstrated that the left hilar mass and the left atrial tumor thrombus had significantly reduced in size, and the symptoms had considerably improved. However, following a 5-month stable period, the patient was hospitalized due to the onset of a fever and severe cough. A CT scan revealed left lower pulmonary atelectasis and pneumonia (Fig. 1C). Mycobacterium tuberculosis was also detected in the sputum.

During the next 6 months, the patient received anti-tuberculosis (300 mg isoniazid; $600 \mathrm{mg}$, rifampicin; daily) and supportive treatment (228 $\mathrm{mg}$ polyene phosphatidylcholine; three times daily). A follow-up CT scan at 1 year post-radiotherapy demonstrated that the tumor thrombus in the left atrium had disappeared, however, the left lower pulmonary atelectasis had not yet been fully alleviated (Fig. 1D). The patient exhibited no marked symptoms, and following completion of a PET scan, there was no indication of tumor recurrence. Despite this, 6 months later, the patient presented with progressive back pain, and a subsequent magnetic resonance imaging scan confirmed the presence of multiple bone metastases. The patient accepted analgesic therapy only and succumbed 3 months later. The patient's overall survival time was 21 months.
Written informed consent was obtained from the family of the patient for the publication of this case report and any accompanying images.

\section{Discussion}

Lung cancer presenting with a giant atrial tumor thrombus is particularly rare. To the best of our knowledge, there have only been 18 cases published in the English literature over the last two decades. In these 18 cases, squamous cell carcinoma was the most common pathological type and was present in 5 cases $(7,9-12)$, followed by 4 neuroendocrine carcinoma cases (13-16), 3 adenocarcinoma cases $(1,17,18), 3$ sarcoma cases $(5,6,19), 1$ large cell carcinoma case (20) and 1 neuroectodermal tumor case (21). The pathology of a further case was reported as sarcoma mixed with squamous carcinoma (16); although histopathological examination demonstrated that the primary tumor was poorly-differentiated, the cardiac and intravascular portions of each tumor were less differentiated and more necrotic than the primary focus in the lung (3).

The morphological style of lung cancer invasion into the left atrium may be divided into two groups: i) Contiguous invasion by the primary tumor from the pulmonary vein base; and ii) direct invasion by the primary tumor, or lymph node metastasis, into the posterior wall of the left atrium (10). The atrial wall is composed of the epicardium, a muscle layer and the tunica intima. As a thin layer of mesothelial cells, the epicardium is easily penetrated by tumor cells with involvement of the muscle layer. Once the tumor cells have invaded the atrium, this alters the hemodynamics of the body; cancer cell-derived microparticles aggregate platelets via a tissue factor-dependent pathway, and as a result, the tumor thrombus rapidly increases in size (22). Tumor thrombi may lead to widespread systemic embolization and/or outflow tract obstruction (4-6). The incidence of embolization is $~ 16.7 \%$ (3/18), and as a result, treatment is always warranted.

Lung cancer invading the left atrium or great vessels is classified as T4 and belongs to stage IIIb of the tumor-node-metastasis 
classification system (8). The prognosis for affected patients is poor, and surgery often has an insufficient effect on the disease. However, patients presenting with limited invasion of the left atrium and no distant metastasis may achieve a complete surgical resection despite having a T4 classification (23). Prior to resection of the mass, a cardiopulmonary bypass should be performed to prevent systemic seeding or embolization of the tumor $(10,16)$. The median survival time following this type of surgery has been reported to be 10 months (24).

Radiotherapy is an important treatment for malignant tumors. In a previous case, intensity-modulated radiotherapy (50-Gy dose) was delivered to a lung tumor and intra-atrial lesion, with the patient experiencing no acute side effects from the radiation and a complete response of the intracardiac disease within 3 months (1). Modern radiation oncology techniques, including stereotactic and intensity-modulated radiotherapy, have greatly reduced the risk of radiation-associated heart disease by delivering a high dose directly to the tumor, and a sharp dose-gradient to the adjacent normal heart and other organs $(1,25)$. Furthermore, due to the increased precision of modern radiotherapy, a smaller margin is applied to the gross tumor volume to overcome daily set-up or tumor motion uncertainty. In particular, the risk of pericardial and coronary artery disease is significantly limited with strategies such as a deep inspiratory breath hold. Improved dose-delivery techniques also increase the utility of radiation $(1,25)$. The 18 previous cases $(1,5-7,9-21)$ show that surgery combined with chemotherapy is the most common treatment for this condition, however, prognosis remains extremely poor, with overall survival times ranging between 4 and 17 months. In the present case, surgery was not advised due to the presence of distant metastasis and the high risk of the surgical procedure. However, a complete response of the tumor and the associated thrombus was achieved using stereotactic radiotherapy alone. However, the patient later suffered from pneumonia and tuberculosis as a result of decreased immunity, which subsequently led to left pulmonary atelectasis. The overall survival time of the patient was 21 months, and no radiation therapy-associated complications were noted. Therefore, the present case demonstrates that stereotactic radiotherapy may be a beneficial palliative treatment for patients with stage IV lung cancer invading the left atrium.

\section{Acknowledgements}

The authors would like to thank Dr Yihong Li and Dr Chunli Liu (Department of Nuclear Medicine, Wujing Hospital) for providing imaging data and Dr Lina Liu (Department of Pathology, Wujing Hospital) and Dr Yanchun Ma (Department of Pathology, Huashan Hospital, Shanghai, China) for performing histopathological analysis.

\section{References}

1. Lee $\mathrm{P}$ and Kishan AU: Radiotherapy is effective for a primary lung cancer invading the left atrium. BMJ Case Rep 2012, 2012.

2. Takahashi K, Furuse M, Hanaoka H, Yamada T, Mineta M, Ono H, Nagasawa K and Aburano T: Pulmonary vein and left atrial invasion by lung cancer: Assessment by breath-hold gadolinium-enhanced three-dimensional MR angiography. J Comput Assist Tomogr 24: 557-561, 2000.
3. Kodama K, Doi O and Tatsuta M: Unusual extension of lung cancer into the left atrium via the pulmonary vein. Int Surg 75: 22-26, 1990.

4. Sadat U, Noor N, See TC and Varty K: Peripheral arterial ischemia by a primary lung tumour invading left atrium. Lung Cancer 57: 237-239, 2007.

5. Koh TW: Invasion of lung mesenchymal chondrosarcoma into the left atrium via the pulmonary vein detected on transoesophageal echocardiography. Eur J Echocardiogr 12: 556, 2011.

6. Woodring JH, Bognar B and van Wyk CS: Metastatic chondrosarcoma to the lung with extension into the left atrium via invasion of the pulmonary veins: Presentation as embolic cerebral infarction. Clin Imaging 26: 338-341, 2002.

7. Shimizu J,Ikeda C, Arano Y, Adachi I,Morishita M, Yamaguchi S, Ishikawa N, Watanabe $\mathrm{G}$ and Minato $\mathrm{H}$ : Advanced lung cancer invading the left atrium, treated with pneumonectomy combined with left atrium resection under cardiopulmonary bypass. Ann Thorac Cardiovasc Surg 16: 286-290, 2010.

8. Tsim S, O'Dowd CA, Milroy R and Davidson S: Staging of non-small cell lung cancer (NSCLC): A review. Respir Med 104: 1767-1774, 2010.

9. Sengül C, Sünbül A, Ozveren O and Değertekin M: Case images: Squamous cell lung cancer metastasis in the left atrium: An interesting case with cardiac images. Turk Kardiyol Dern Ars 40: $654,2012$.

10. Ueda K, Kaneda Y, Sakano H, Tanaka T, Saito K and Hamono K: Successful treatment of intracardiac progression and metachronous multiple brain metastases from primary lung cancer. Jpn J Thorac Cardiovasc Surg 54: 168-170, 2006.

11. Kim JH, Jung JY, Park Y, Hwang SI, Jung CS, Lee SH and Yoo CW: Non-small cell lung cancer initially presenting with intracardiac metastasis. Korean J Intern Med 20: 86-89, 2005.

12. Asaad K, Sadaba JR and Nair RU: Intracardiac extension of squamous cell carcinoma of lung. Eur J Cardiothorac Surg 24: 640, 2003.

13. Costache VS, Lantuejoul S, Stoica S, Fluttaz A, Hacini R and Brichon PY: Giant intracardiac neoplasic thrombus of a large cell neuroendocrine carcinoma of the lung. Cardiovasc Pathol 19: e85-e87, 2010.

14. Lin MT, Ku SC, Wu MZ and Yu CJ: Intracardiac extension of lung cancer via the pulmonary vein. Thorax 63: 1122, 2008.

15. Brandt RR, Rubin J and Reeder GS: Intracardiac extension of a lung tumor causing left ventricular inflow obstruction. J Am Soc Echocardiogr 8: 930-933,1995.

16. Ma Q, Liu D, Liu P, Chen J, Xie Z and D'Amico TA: Extensive invasion of the left atrium by lung cancer. Ann Thorac Surg 96: 685-687, 2013.

17. Alexandrescu C, Civaia F and Dor V: Tumor thrombus in right atrium from lung adenocarcinoma. Ann Thorac Surg 87: e11-e12, 2009.

18. Ucak A, Inan K, Onan B, Temizkan V, Alp I and Yilmaz AT: Free-floating tumor thrombus in the left atrium associated with non-small cell lung cancer. J Card Surg 24: 686-689, 2009.

19. Dogan A, Icli A, Arslan A, Varol E and Ozaydin M: Metastatic atrial sarcoma extending from the lung into the left atrium via a pulmonary vein. Exp Clin Cardiol 17: 77-78, 2012.

20. Khan N, Golzar J, Smith NL and Movahed A: Intracardiac extension of a large cell undifferentiated carcinoma of lung. Heart 91: 512, 2005.

21. Yokouchi H, Kodama K, Higashiyama M, Takami K, Kobayashi T, Takami H, Nakamura S and Horai T: Successful removal of a primitive neuroectodermal tumor in the lung with gross extension into the left atrium. Thorac Cardiovasc Surg 47: 257-259, 1999.

22. Thomas GM, Panicot-Dubois L, Lacroix R, Dignat-George F, Lombardo D and Dubois C: Cancer cell-derived microparticles bearing P-selectin glycoprotein ligand 1 accelerate thrombus formation in vivo. J Exp Med 206: 1913-1927, 2009.

23. DiPerna CA and Wood DE: Surgical management of T3 and T4 lung cancer. Clin Cancer Res 11: 5038s-5044s, 2005.

24. Fukuse T, Wada $\mathrm{H}$ and Hitomi S: Extended operation for non-small cell lung cancer invading great vessels and left atrium. Eur J Cardiothorac Surg 11: 664-669, 1997.

25. Remouchamps VM, Vicini FA, Sharpe MB, Kestin LL, Martinez AA and Wong JW: Significant reductions in heart and lung doses using deep inspiration breath hold with active breathing control and intensity-modulated radiation therapy for patients treated with locoregional breast irradiation. Int J Radiat Oncol Biol Phys 55: 392-406, 2003. 